\title{
Formation continue au top niveau: pourquoi ai-je besoin d'un diplôme de formation continue?
}

Christoph Hänggeli ${ }^{a}$,

Werner Bauer ${ }^{b}$, Ernst Gähler ${ }^{c}$

a directeur de l'ISFM

b président de l'ISFM

c vice-président de la FMH
Correspondance:

Institut suisse pour la formation médicale postgraduée et continue (ISFM) Elfenstrasse 18 CH-3000 Berne 15 Tél. 0313591111 Fax 0313591112

siwf[at]fmh.ch
La plate-forme de formation continue en ligne de l'ISFM permet aux médecins de saisir facilement la formation continue accomplie et d'imprimer eux-mêmes le diplôme correspondant dès qu'ils ont rempli les conditions du programme de formation continue. La procédure est également simplifiée pour les sociétés de discipline médicale: elles peuvent contrôler sans peine les données saisies de façon électronique puis approuver l'impression du diplôme. Les diplômes de formation continue sont automatiquement publiés sur la plate-forme www.doctorfmh.ch, ce qui rend obsolète toute attestation individuelle vis-àvis des autorités sanitaires et des assureurs-maladie. La charge administrative s'en trouve ainsi réduite à un minimum, tant pour les sociétés de discipline médicale que pour les médecins devant accomplir une formation continue. Les médecins non abonnés à HIN pourront égale- ment se connecter à cette plate-forme avec un nom d'utilisateur et un mot de passe à partir de septembre 2012.

Les assureurs continuent de rémunérer les prestations de droits acquis aux médecins dont le diplôme de formation continue est publié sur www.doctorfmh.ch. Pour cette raison, les sociétés qui ne sont pas encore connectées à la plate-forme de formation continue en ligne de l'ISFM doivent, dans l'intérêt de leurs membres, envoyer régulièrement à l'ISFM une liste Excel contenant tous les diplômes de formation continue délivrés.

\section{La formation continue: un devoir profession- nel réglementé par la loi}

Pour toutes les cinq professions médicales universitaires, l'obligation d'effectuer une formation continue est prévue explicitement en tant que devoir pro-

\section{La formation continue en 10 points}

1. Le devoir de formation continue est un devoir professionnel ancré dans l'art. 40 de la loi sur les professions médicales (LPMéd). La surveillance et les contrôles incombent aux autorités cantonales concernées.

2. L'ISFM offre un diplôme de formation continue conjointement avec les 45 sociétés de discipline médicale représentant un titre de spécialiste. Ce diplôme

- atteste la formation continue accomplie selon la LPMéd;

- valide les positions de droits acquis.

3. Conformément à la Réglementation pour la formation continue (RFC), les médecins doivent acquérir au moins un diplôme de formation continue dans le domaine correspondant à leur activité professionnelle principale du moment.

4. Chaque programme de formation continue des 45 sociétés de discipline médicale fait la distinction entre les catégories suivantes:

- formation continue essentielle (25 crédits)

- formation continue élargie (25 crédits)

- travail personnel (30 heures, non contrôlées)

La formation continue obligatoire comprend ainsi 80 heures de formation continue par an. Les médecins en activité doivent attester 150 crédits (dont au moins 75 crédits de formation continue essentielle) tous les trois ans.

5. Les médecins qui facturent des positions de droits acquis dans le cadre du TARMED doivent suivre une formation continue aussi dans ces domaines, conformément au concept de la valeur intrinsèque. La formation continue pour ces positions peut être accomplie dans le cadre de la formation continue élargie avec 75 crédits sur une période de 3 ans.

6. Le diplôme de formation continue doit être renouvelé tous les 3 ans.

7. Si vous n'acquérez pas (ne voulez pas acquérir) un diplôme de formation continue, vous avez l'obligation de déclarer la formation continue pour les prestations de droits acquis sur myfmh, et ce pour chaque position (www.myfmh.ch).

8. La recertification (formation continue) d'attestations de formation complémentaire a lieu d'après le programme de formation complémentaire concerné et elle est indépendante des 45 diplômes de formation continue des titres de spécialiste. La non-observation des règles de recertification peut entraîner la perte de l'attestation.

9. Vous trouverez sur notre site internet www.siwf.ch, à la rubrique «Formation continue», les 45 programmes de formation continue, les interlocuteurs des sociétés de discipline médicale concernées et toutes les autres informations.

10. La plate-forme de formation continue (www.siwf.ch $\rightarrow$ Formation continue $\rightarrow$ Plate-forme de formation continue) vous permet d'enregistrer continuellement vos activités et d'imprimer vous-même le diplôme de formation continue après l'obtention des 150 crédits exigés (uniquement pour les disciplines répertoriées). 
fessionnel dans la loi sur les professions médicales (LPMéd). Les autorités cantonales chargées de la surveillance peuvent sanctionner tout manquement au devoir de formation continue par un avertissement ou une amende pouvant aller jusqu'à 20000 francs. En revanche, la mise en œuvre de ce devoir légal, notamment son ampleur et ses modalités, incombe aux organisations professionnelles. Conjointement avec les sociétés de discipline médicale, l'ISFM propose au corps médical un diplôme de formation continue reconnu de la même façon par les autorités sanitaires et les assureurs-maladie. Il existe dans chaque domaine (uniquement titres de spécialiste) un programme de formation continue qui règle en détail les conditions d'obtention du diplôme. Le choix parmi les 45 programmes de formation continue re-

Figure 1

Structure des 80 heures de formation continue exigées par année.

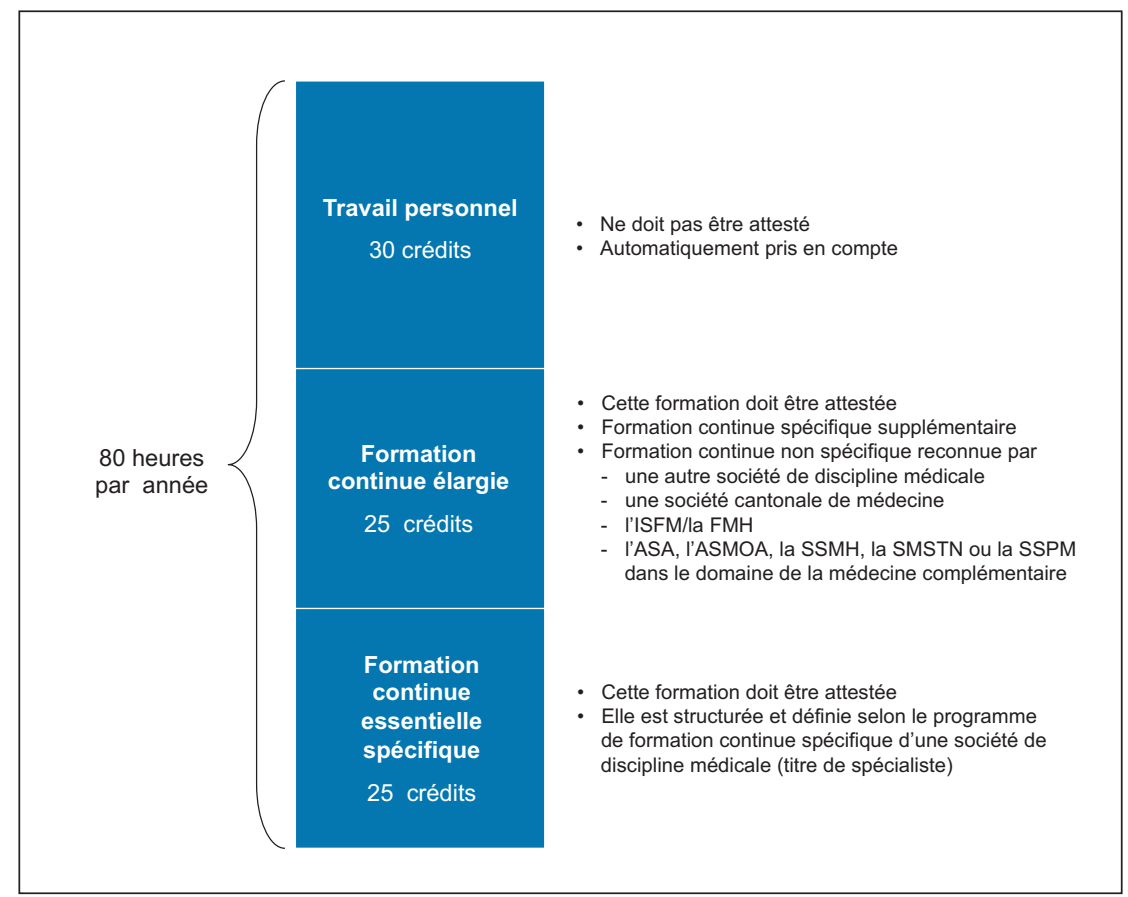

Figure 2

Page d'accès à la plate-forme de formation continue.

\begin{tabular}{|c|c|}
\hline \multicolumn{2}{|c|}{$\begin{array}{l}\text { SIWF } \\
\text { ISFM }\end{array}$} \\
\hline 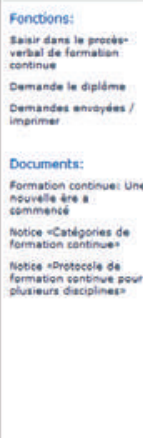 & 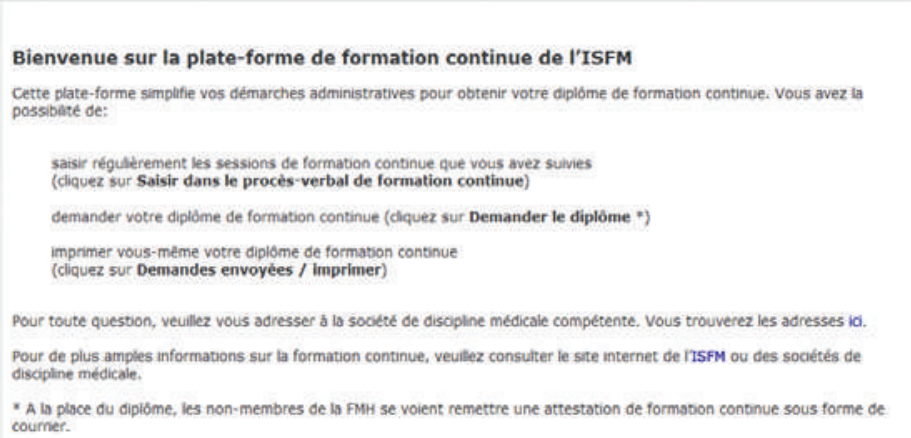 \\
\hline & $\Theta$ internet| Geschoutzer Modus Inakiv \\
\hline
\end{tabular}

lève de la responsabilité de chacun. Il est recommandé de choisir un programme qui correspond le plus à l'activité professionnelle principale du moment. Le devoir de formation continue commence dans l'année suivant l'obtention du titre de spécialiste ou le début de l'activité médicale en Suisse. Les médecins qui se trouvent en formation postgraduée en vue d'un titre de spécialiste ou de formation approfondie ne sont pas soumis à cette exigence.

\section{Formation continue essentielle spécifique et formation continue élargie}

Le nombre d'heures de formation continue conseillé par an (fig. 1) est de 80 heures (= crédits). Les médecins doivent attester 50 crédits par an ou 150 crédits au cours d'une période de formation continue de trois ans. Chaque programme définit une formation continue essentielle spécifique de 25 crédits par an. Dans ce contexte, les sociétés de discipline médicale peuvent définir différentes catégories de formation et fixer le nombre maximal de crédits acceptés par catégorie. Les médecins peuvent faire valider 25 crédits au plus en tant que formation continue élargie, ce qui leur permet de suivre une formation continue hors de leur discipline principale. On entend par formation continue élargie, à côté d'une formation essentielle spécifique supplémentaire, toutes les sessions de formation pour lesquelles des crédits sont octroyés par une autre société de discipline médicale, une société cantonale de médecine ou l'ISFM. Les questions relatives au devoir de formation continue dans une discipline précise doivent être adressées à la société concernée qui saura y répondre.

\section{Comment puis-je obtenir le diplôme de formation continue?}

La plate-forme de formation continue de l'ISFM (www.isfm.ch $\rightarrow$ Formation continue; cf. fig. 2) est la voie la plus simple pour obtenir le diplôme de formation continue. Cette plate-forme vous permet d'enregistrer continuellement vos activités dans un protocole personnel et d'imprimer vous-même votre diplôme au terme de la période de formation continue de trois ans - à condition bien sûr d'avoir obtenu les 150 crédits exigés et de les avoir fait valider par la so-

Droits acquis sous stricte surveillance des assureurs Les assureurs ont annoncé qu'ils ne prendront désormais en charge plus que les positions de droits acquis validées. Elles le deviennent automatiquement à l'obtention d'un diplôme de formation continue par la plate-forme de formation continue de l'ISFM. Les sociétés qui ne sont pas connectées à cette plate-forme doivent communiquer à l'ISFM les diplômes délivrés en vue de leur publication dans la liste des médecins sur www.doctorfmh.ch et pour le maintien des positions de droits acquis. Tant qu'un diplôme de formation continue ne figure pas dans cette liste, les médecins ont la possibilité de faire valider leurs droits acquis sous www.myfmh.ch. Dans ce cas, ils doivent attester la formation continue pour chaque position en particulier. 
Figure 3

Exemple de saisie sur doctorfmh.

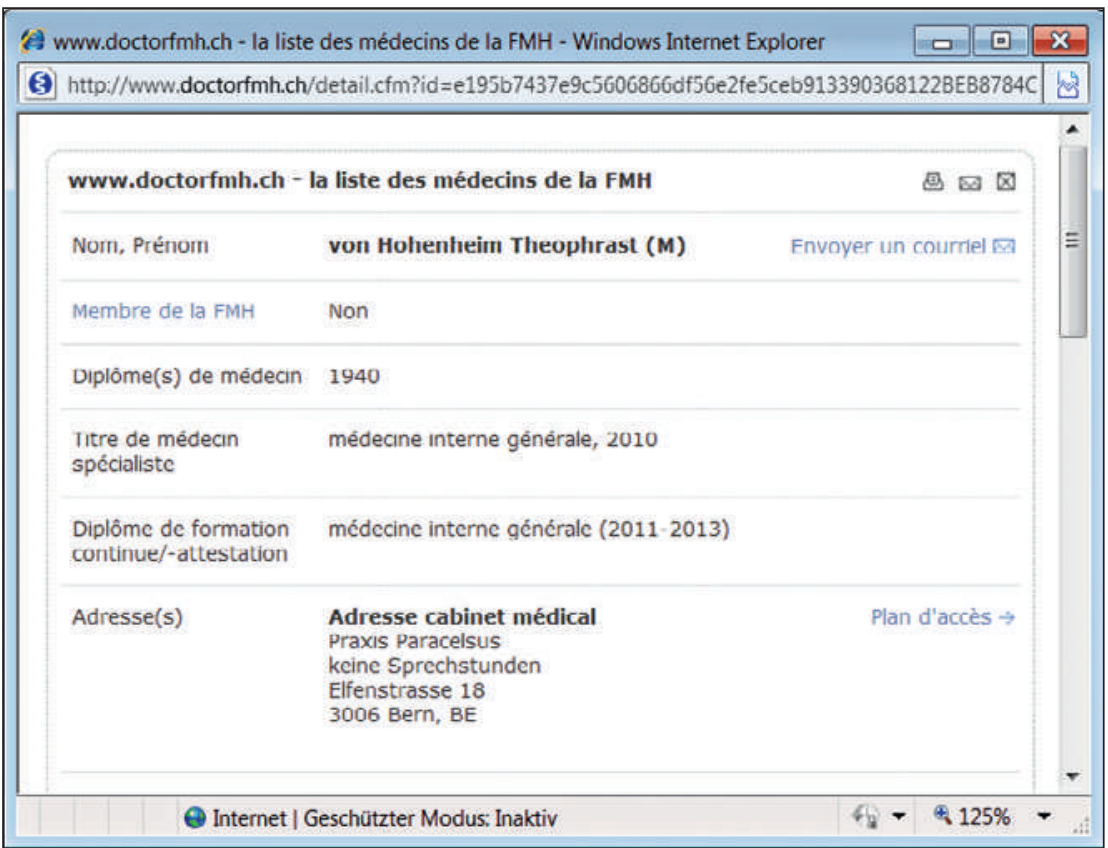

ciété concernée. Cependant, toutes les sociétés ne sont pas encore connectées à la plate-forme de formation continue. Dans ce cas, le médecin doit contacter la société dont il souhaite acquérir le diplôme de formation continue. Inconvénient: les diplômes de formation continue ne sont pas automatiquement publiés sur www.doctorfmh.ch, mais ils doivent être communiqués à l'ISFM par la société au moyen d'une liste Excel et saisis dans la liste des médecins pour pouvoir être pris en considération. Ces diplômes sont exclusivement destinés aux membres de la FMH. Les non-membres obtiennent une attestation équivalente certifiant à l'intention des autorités sanitaires et des assureurs-maladie que le devoir de formation continue a été accompli.

\section{Pas de rémunération des prestations de droits acquis sans diplôme de formation continue!}

Dix ans après l'entrée en vigueur du TARMED, 11000 médecins font encore valoir 765000 prestations de droits acquis. Pour pouvoir continuer à les facturer, ils doivent attester leur formation continue au moyen d'un diplôme approprié. Pour cela, ils disposent des programmes de formation continue des 45 titres de spécialiste. Grâce à la «formation continue élargie», chaque programme offre la possibilité de suivre une formation continue dans les disciplines de chacune des positions sollicitées. Ces positions sont automatiquement validées et prolongées conformément à la durée de validité du diplôme de formation continue.

\section{Conclusion}

Pour les médecins conscients de leurs responsabilités, effectuer une formation continue tout au long de la vie professionnelle est une évidence. La plate-forme en ligne et le diplôme de formation continue leur permettent d'attester sans autre formalité que leurs compétences professionnelles sont au top niveau. Un diplôme, deux fonctions: la preuve du devoir légal accompli est fournie et toutes les positions de droits acquis sont validées. Pourquoi ne pas demander dès aujourd'hui votre diplôme de formation continue?
Diplômes de formation continue dans la liste des médecins www.doctorfmh.ch
La liste des médecins www.doctorfmh.ch contient, outre toutes les qualifications professionnelles offi- cielles selon la Réglementation pour la formation post- graduée (RFP), également les diplômes de formation continue avec leur période de validité (cf. fig. 3). En re- vanche, elle ne contient pas d'autres informations, par exemple concernant les sessions enregistrées dans le protocole, afin de garantir la protection des données.

1 Hänggeli C. Possédez-vous un diplôme de formation continue? Bull Méd Suisses. 2009;90(49):1906-8.

\section{Questions fréquentes}

1. Vais-je perdre mon titre de spécialiste si je ne fais pas de formation continue?

Non, la formation continue est un devoir professionnel dont le manquement peut être sanctionné par les autorités sanitaires cantonales par un avertissement ou une amende pouvant aller jusqu'à 20000 francs. Le retrait du titre de spécialiste n'est pas possible.

2. Qui a l'obligation d'accomplir la formation continue?

En vertu de l'art. 9 de la Réglementation pour la formation continue (RFC), tous les détenteurs d'un titre postgrade fédéral (aussi celui de «Médecin praticien») ou d'un titre postgrade étranger reconnu sont tenus d'accomplir une formation continue aussi longtemps qu'ils exercent une activité médicale en Suisse. Les médecins qui se trouvent en formation postgraduée en vue d'un titre de spécialiste ou de formation approfondie ne sont pas soumis à cette exigence, ainsi que les médecins en formation qui possèdent déjà un titre de formation postgraduée.

3. Je me rends aux Etats-Unis pour travailler deux ans dans la recherche. Suis-je tenu d'accomplir une formation continue? Non, vous n'êtes pas tenu d'accomplir une formation continue pendant cette période. Le devoir de formation continue s'applique uniquement aux médecins exerçant une activité médicale en Suisse (art. 9 RFC). Le devoir de formation continue ne reprendra vigueur qu'à votre retour en Suisse. Vous pouvez soit commencer une nouvelle période de formation continue de trois ans, soit faire valoir une réduction de deux ans de la formation continue obligatoire pour votre séjour à l'étranger. Il faut préciser que les séjours de courte durée à l'étranger (inférieurs à un an) ne suffisent pas pour suspendre le devoir de formation continue.

4. Je travaille dans le secteur administratif de Swissmedic en tant que médecin porteur d'un titre de spécialiste en médecine interne générale. Suis-je tenu d'accomplir une formation continue? 
Le devoir de formation continue concerne tous les médecins qui exercent une «activité médicale». Une telle activité existe lorsque vous examinez, conseillez ou soignez des patients sous une forme quelconque. Une activité administrative ou de recherche sans contact avec des patients n'entre pas dans cette catégorie.

5. Que se passe-t-il si je n'acquiers pas un diplôme de formation continue?

L'acquisition du diplôme de formation continue n'est pas une obligation stipulée par la loi. L'important est que vous accomplissiez autant de formation continue que prescrit. Cela ne concerne en aucun cas votre titre de spécialiste. Ne pas posséder de diplôme de formation continue entraîne toutefois les inconvénients suivants:

- en cas de contrôle, vous devez convaincre les autorités cantonales concernées que la formation continue accomplie correspond au standard habituel. II en va de même lors d'un procès éventuel de responsabilité civile.

- en l'absence de diplôme ou d'attestation de formation continue dans le registre doctorfmh.ch, vous devez confirmer dans la banque de données (www.myfmh.ch) la formation continue spéciale pour les prestations de droits acquis et l'attester au moyen de documents adéquats [1]. Si vous ne le faites pas, les assureurs peuvent refuser la prise en charge des positions de droits acquis.

6. Quel est le coût d'un diplôme de formation continue?

La reconnaissance de sessions de formation continue et le contrôle de la formation continue sont effectués par la société de discipline médicale concernée, laquelle perçoit des taxes en fonction des dépenses occasionnées. Pour les membres d'une société, cette taxe est généralement incluse dans la cotisation annuelle. Pour les nonmembres, le diplôme valable pour trois ans ne devrait pas coûter plus de 150 francs. Des taxes plus élevées devront être approuvées par le Comité de I'ISFM.

7. Comment puis-je accomplir une formation continue dans le cadre de la médecine complémentaire si ma société ne reconnaît aucune session de formation correspondante?

Dans la Réglementation pour la formation continue, la médecine complémentaire fait l'objet d'une disposition spéciale: les cinq sociétés qui gèrent une attestation de formation complémentaire (ASA, ASMOA, SSMH, SMSTN, SSPM) peuvent reconnaître des sessions de formation et octroyer des crédits correspondants qu'il est possible de faire valider dans le cadre de la formation continue élargie.

8. Puis-je faire valoir la formation continue que j'accomplis dans le cadre d'une attestation de formation complémentaire également pour le diplôme de formation continue?

Seules les sessions de formation pour lesquelles des crédits sont octroyés par une société de discipline médicale (titres de spécialiste), une société cantonale de médecine, les 5 sociétés de médecine complémentaire (ASA, ASMOA, SSMH, SMSTN, SSPM) ou I'ISFM peuvent être prises en compte pour le diplôme de formation continue. Les sessions de formation d'autres organisations médicales doivent être reconnues au moins par une société de discipline médicale. Lorsque c'est le cas, ces sessions peuvent être prises en compte dans le cadre de la formation continue élargie pour les 44 autres diplômes de formation continue.

9. Je suis porteur du titre de spécialiste en médecine interne générale et j'exerce principalement dans le domaine gynécologique. Je dispose des positions de droits acquis correspondantes. Puis-je acquérir le diplôme de formation continue de la Société suisse de gynécologie et d'obstétrique (SSGO) et attester ainsi la formation continue pour les prestations de droits acquis?

Comme vous exercez principalement dans le domaine de la gynécologie et de l'obstétrique, vous avez intérêt à choisir le programme de formation continue de la SSGO. Mais en vertu de l'art. 12 de la RFC, le diplôme de formation continue n'est remis qu'aux porteurs du titre correspondant. Si vous validez 150 crédits, vous avez droit à une attestation de formation continue équivalente qui vous servira entre autres à attester la formation continue requise pour les droits acquis.

10. Je possède deux titres de spécialiste, I'un en médecine interne générale et l'autre en cardiologie. Dois-je obtenir le diplôme de formation continue dans ces deux domaines?

Non. Vous pouvez vous limiter au programme de formation continue qui correspond le plus à l'activité professionnelle que vous exercez. Vous êtes bien évidemment libre de suivre deux programmes de formation continue. Cette double formation n'entraîne pas une importante surcharge de travail puisque la formation continue essentielle d'un des programmes est automatiquement considérée comme la formation continue élargie de l'autre. Dès que vous avez accompli les formations continues essentielles spécifiques des deux disciplines sans chevauchement, vous avez droit aux deux diplômes de formation continue.

11. Je suis porteur d'un titre de spécialiste en médecine interne générale avec une formation approfondie en gériatrie. Existe-il un diplôme de formation continue pour cette spécialisation?

Non. Depuis la révision de la Réglementation pour la formation continue du 26 mai 2010, seuls 45 programmes de formation continue sont proposés, soit un par titre de spécialiste. Cependant, la formation continue en gériatrie est prise en compte sans limitation de contenu pour le diplôme de formation continue en médecine interne générale.

12. Je suis mère de deux enfants et je travaille à 50\% dans un cabinet médical. Dois-je accomplir l'intégralité de la formation continue?

Oui. Un taux d'occupation partiel ne donne pas droit à une réduction de la formation continue obligatoire. La formation continue a pour objectif de garantir la qualité des prestations et de maintenir la compétence médicale, une garantie qu'il convient d'apporter également lors d'une activité à temps partiel.

13. J'ai interrompu mon activité médicale pendant un congé maternité de six mois. Dois-je valider moins de crédits pour le diplôme de formation continue?

Oui. Des interruptions de l'activité médicale dépassant six mois dans la période de formation de trois ans entraînent une réduction pro rata des crédits exigés. Ce même principe s'applique aux séjours à l'étranger ou à d'autres interruptions de l'activité médicale en Suisse.

14. Je suis porteur du titre de «Médecin praticien». Quelle formation continue dois-je effectuer? Les porteurs du titre de «Médecin praticien» peuvent faire leur choix parmi les 45 domaines répertoriés. Nous leur recommandons de choisir le programme de formation continue correspondant à l'activité professionnelle exercée sur le moment. 